\title{
Automatic extraction of faults and fractal analysis from remote sensing data
}

\author{
R. Gloaguen ${ }^{1}$, P. R. Marpu ${ }^{1}$, and I. Niemeyer ${ }^{2}$ \\ ${ }^{1}$ Institute for Geology, Freiberg University of Mining and Technology, 09599 Freiberg, Germany \\ ${ }^{2}$ Institute for Mine-Surveying and Geodesy, Freiberg University of Mining and Technology, 09599 Freiberg, Germany
}

Received: 18 December 2006 - Revised: 23 February 2007 - Accepted: 3 March 2007 - Published: 22 March 2007

\begin{abstract}
Object-based classification is a promising technique for image classification. Unlike pixel-based methods, which only use the measured radiometric values, the objectbased techniques can also use shape and context information of scene textures. These extra degrees of freedom provided by the objects allow the automatic identification of geological structures. In this article, we present an evaluation of object-based classification in the context of extraction of geological faults. Digital elevation models and radar data of an area near Lake Magadi (Kenya) have been processed. We then determine the statistics of the fault populations. The fractal dimensions of fault dimensions are similar to fractal dimensions directly measured on remote sensing images of the study area using power spectra (PSD) and variograms. These methods allow unbiased statistics of faults and help us to understand the evolution of the fault systems in extensional domains. Furthermore, the direct analysis of image texture is a good indicator of the fault statistics and allows us to classify the intensity and type of deformation. We propose that extensional fault networks can be modeled by iterative function system (IFS).
\end{abstract}

\section{Introduction}

The object-based classification requires a segmentation of remote sensing data into highly homogeneous regions (or objects). These image segments correspond to approximations of real world objects, characterised by shape and texture (Benz et al., 2004). Often, in geological image processing, the objects (e.g. volcanoes, rivers, faults, etc) to be identified in the image can be visually separated based on their shape and texture details. This paper details an efficient application of object-based classification of geological features,

Correspondence to: R. Gloaguen

(gloaguen@geo.tu-freiberg.de) which involves the extraction of normal fault morphology for further statistical analysis.

A rift is a region where the Earth's crust is under extensional strain, hence forming a series of horsts and grabens bounded by faults. The fractures generated in this process of rifting are dominantly normal faults. Figure 1 shows the geometry in a rift.

The study area is located in the vicinity of Lake Magadi in Southern Kenya (Fig. 2). The N10 trending elongated Magadi graben, bounded on both sides by major escarpments which cut Precambrian basement, is partly filled by trachytes dated at 1.6 1.1 Ma (e.g., Baker, 1958; Baker et al., 1988). Fault orientation, striation and focal mechanism analysis (Gloaguen et al., 1999; Gloaguen, 2000), kinematic models (Chu and Gordon, 1999) and a recent plate velocity model (Sella et al., 2002, REVEL) have shown that the stress field remained stable in intensity and direction during the last $4 \mathrm{Ma}$ with $\sigma 3$ approximately $\mathrm{EW}$ and an extension rate of about $4 \mathrm{~mm} \mathrm{yr}^{-1}$. The crust, whose thickness is about $35 \mathrm{~km}$, shows a typical continental velocity structure with high velocity lenses at its base interpreted as mafic underplating (e.g., Mechie et al., 1994; Birt et al., 1997). The effective elastic thickness determined from gravity coherence analyses is $15 \pm 4 \mathrm{~km}$, comparable to the seismogenic layer thickness of $18 \mathrm{~km}$ (Gloaguen, 2000). The study area comprises fault zones with hundreds of recent faults thus allowing their statistical analysis. The Magadi trough is mainly filled by trachytes erupted over a short time period (e.g., Baker, 1958). The faults analysed represent deformation in a "homogenous" layer. The arid climate and minimal erosion in this region have preserved the morphology of the faults.

Algorithms based on the automatic classification procedure given in Marpu et al. (2006) are formulated to process remote sensing data. The results of the algorithms and the further application of the results in determining the statistics of the fault populations using the object concept are discussed. Further, the fractal behaviour is studied on the image texture. 


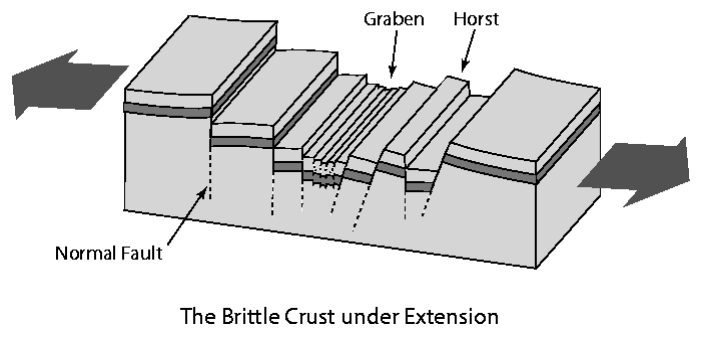

Fig. 1. Fault geometry in a rift.

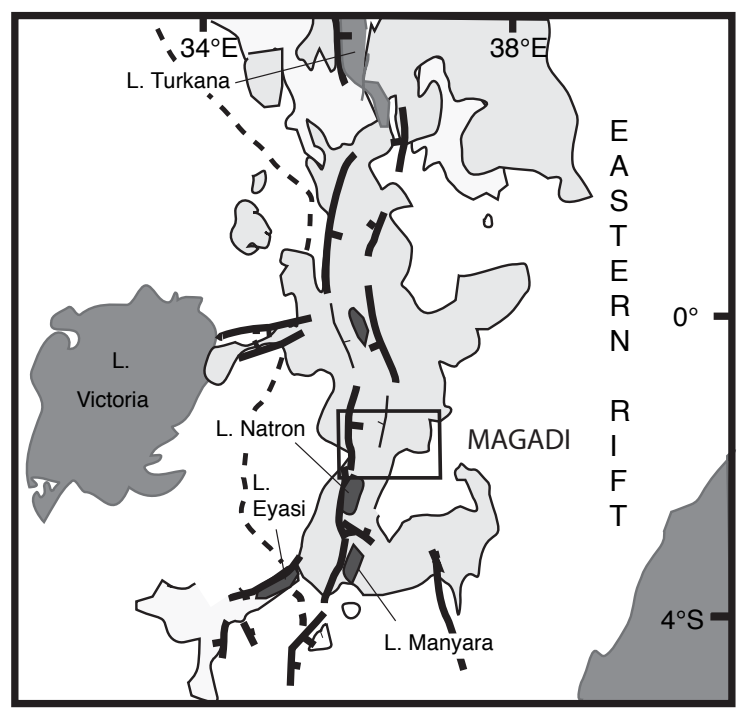

Fig. 2. Location of the working area. Lake Magadi is located in the Southern part of the Kenya Rift. Light grey areas represent PlioQuaternary volcanics, black lines are major rift escarpments.

\section{Data}

We use orthorectified and speckle filtered Radarsat Fine Beam data. Field controls showed that the resolution of the Synthetic Aperture Radar (SAR) is sufficient to measure all of the faults that breach the surface. Discrepancies between fault distribution and statistical laws cannot, therefore, be attributed to the sampling. The radar data are acquired in ascending and descending orbits, allowing to measure precisely faults otherwise in shadow on optic data. By virtue of the geometry of side-looking imaging radar, the same scene imaged in opposite directions can effectively map the faults (Henderson and Lewis, 1998). Most of the faults within the Kenya Rift are oriented N-S and are consequently almost orthogonal to the radar beam. Within such a context, it is obvious that SAR images significantly enhance the mapping of the fault systems compared to optical data. The pre-processing step involves a reduction of the speckle in the SAR data using a Gamma filter.

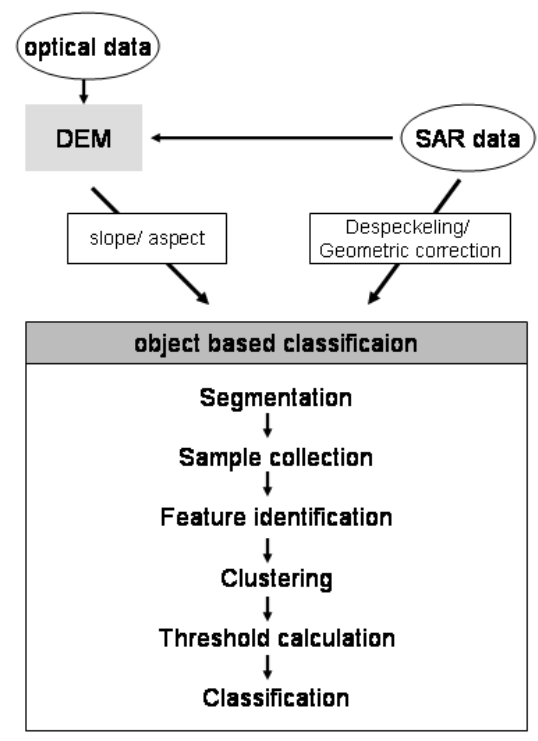

Fig. 3. Schematic procedure for automatic fault extraction.

As the faults are characterised by steep slopes Digital Elevation Model (DEM) can therefore be used for identification. A DEM can be generated by numerous techniques, the most commonly being based on the stereoscopic potential of optical and radar stereo-pairs. Radar data are one of the sources for generating DEMs and hence are the basic input in the process of fault mapping. The pre-processing step here involves generating new image layers from the DEM with the slope and aspect information. The orientation of the pixels in their corresponding neighbourhood gives the direction of the slopes. The gradient of the DEM and the direction information are the input for the algorithm.

\section{Extraction of faults}

The procedure for automatic object-based classification described in Marpu et al. (2006) is used to extract the faults. The algorithm for extracting the morphology of faults is shown in Fig. 3.

The automatic classification procedure starts with segmenting the image (dividing the image in to smaller homogenous regions). Segmentation is the most important step for object-based classification. It is essential for any classification that the approximations (or subsets) of real world objects are first clearly identified within the image. If the segmentation algorithm is not capable of properly segmenting the image, then we cannot expect a proper classification. The image segmentation into primitive objects is done using multi-resolution segmentation algorithm of Definiens Professional $^{1}$ software. The procedure identifies the optimum fea-

\footnotetext{
${ }^{1}$ http://www.definiens.com
} 
tures based on Jeffries-Matusita distance (J). For two classes $C_{1}$ and $C_{2}$ of size $n_{1}, n_{2}$ with means $m_{1}, m_{2}$ and standard deviations $\sigma_{1}, \sigma_{2}$ respectively and assuming gaussian distribution,

$J=2\left(1-e^{-B}\right)$.

where $B$ is the Bhattacharya distance.

$B=\frac{1}{8}\left(m_{1}-m_{2}\right)^{2} \frac{2}{\sigma_{1}^{2}+\sigma_{2}^{2}}+\frac{1}{2} \ln \left(\frac{\sigma_{1}^{2}+\sigma_{2}^{2}}{2 \sigma_{1} \sigma_{2}}\right)$

Based on the identified features which characterise the classes, an approximate distribution is generated using a minimum-distance clustering. Besides determining which features best separate the object classes among each other, it is essential to know which decision threshold allows maximum separability.The approximate distributions are then corrected using the thresholds of separation for every feature to get the final distribution. The thresholds are determined by Bayes' rule as (Nussbaum et al., 2005)

$T=\frac{m_{2} \sigma_{1}^{2}-m_{1} \sigma_{2}^{2} \pm \sigma_{1} \sqrt{\left(\left(m_{1}-m_{2}\right)^{2}+2 A\left(\sigma_{1}^{2}-\sigma_{2}^{2}\right)\right.}}{\left(\sigma_{1}^{2}-\sigma_{2}^{2}\right)}$

where,

$A=\log \left[\frac{\sigma_{1}}{\sigma_{2}} \frac{n_{2}}{n_{1}}\right]$

We calculate the separability and corresponding thresholds for each possible object class combination and for any number of given features. The results are hierarchically ordered according to separability for each object class combination. These in turn enable the compilation of a rule-based classification model.

We first classify directly the acquired radar data (Radarsat Fine Beam). The input parameters for the multi-resolution segmentation algorithm are carefully selected on a master image. These input parameters are normally transferable to the slave images. The result was not as accurate as expected. Fig. 4 shows the classification in one of the images.

Two reasons are identified for this. The first one being the considerable amount of speckle remaining in the image even after a great deal of filtering and the second reason is the large, non-gaussian variability of the image values within the real-world fault objects, which makes it difficult for the segmentation algorithm to clearly identify proper objects. The classification in this case is not highly accurate, but can still be considered for mapping faults. However, a post-classification statistical analysis of faults is not possible with such an approach. We therefore generate radargrammetric DEMs.

The same procedure is then applied on the DEM of the same region. The gradient of the DEM identifies all the slopes (Fig. 5).

The real-world fault object regions are homogenous in the gradient image and hence the multi-resolution segmentation

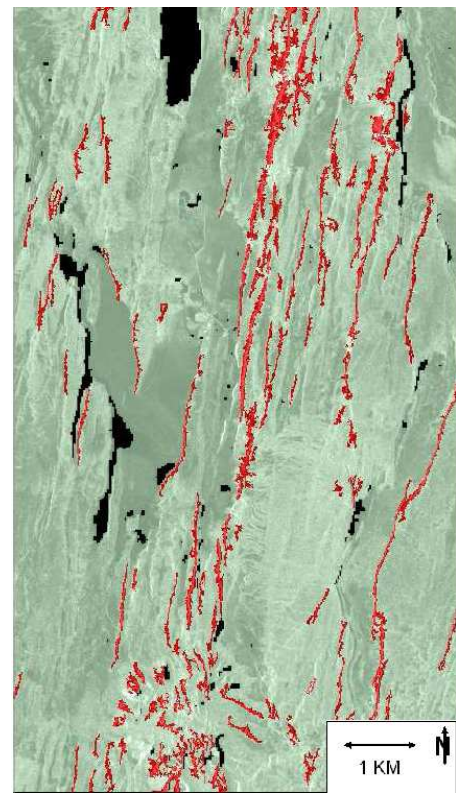

Fig. 4. Extracted faults in a radar image.

algorithm resolves the image into better image objects. Figure 5 shows the classified image. It can be observed that the individual faults are resolved accurately. The two colours indicate the two throw directions of the faults. Disturbing regions such as volcanoes are masked in order to avoid misclassification of volcanic flows as faults.

\section{Fractal analysis}

The distributions of fault dimensions, such as displacement and length, have been used to understand the evolution of fault systems (e.g., Watterson, 1986; Marrett and Allmendinger, 1991; Cowie and Scholz, 1992). These relations have implications for rupture mechanics and dynamics, and they provide links to empirical earthquake scaling relations (e.g., Cowie and Scholz, 1992). Fault-population distributions have been correlated with different statistic laws such as gamma (Cardon, 1999), log-normal (Davy, 1993; Castaing et al., 1995), logarithmic (e.g., Villemin and Sunwoo, 1987), or power-law (e.g., Velde et al., 1990; Velde and Dubois, 1991). Power-law has a specific interest on its own as the exponent is interpreted as the fractal dimension and the faulting has then a fractal behaviour, at least in one of its dimensions.

Objects which display self-similarity over an infinitely extended scale range are fractals and every fractal is characterized by a unique fractal dimension. However, in geology we have to confine ourselves to a finite scale range. At every scale the object appears to have a similar shape in the finite scale range. And the fractal dimension can be defined 

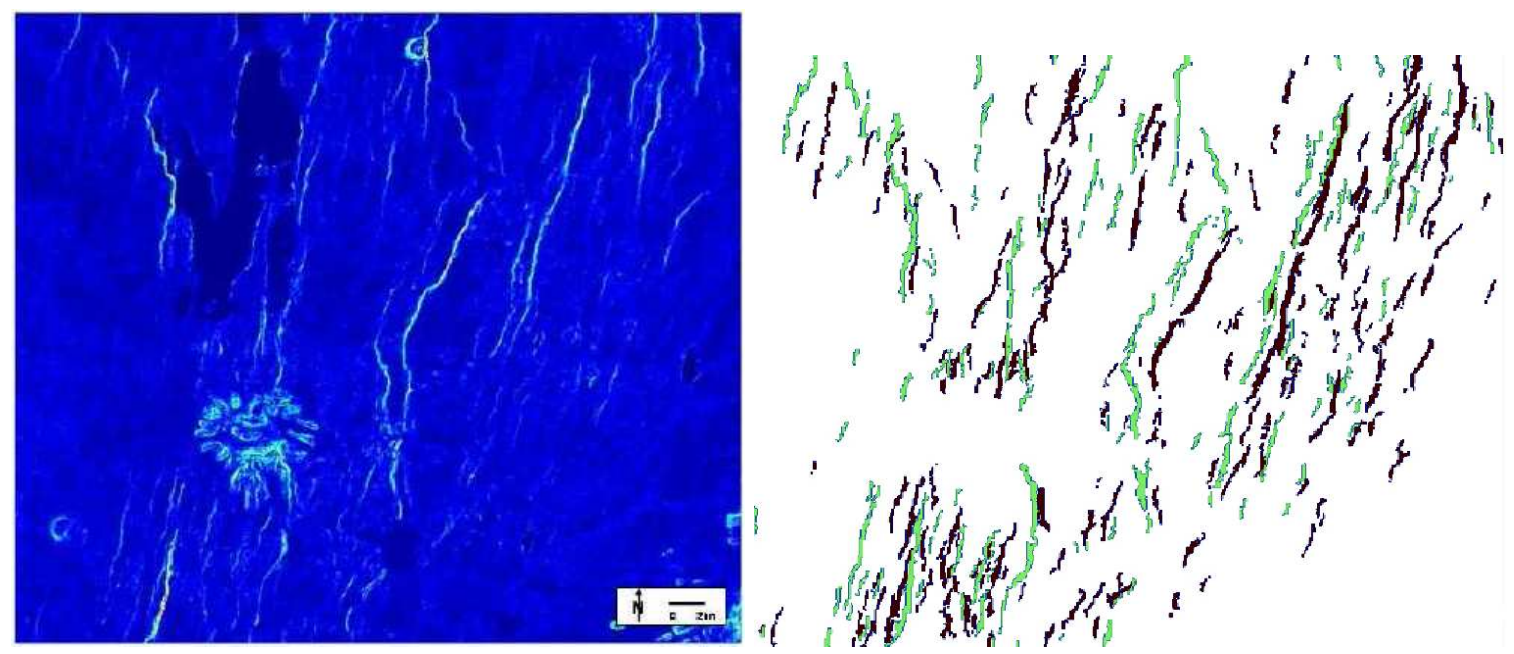

Fig. 5. Derivative of a DEM enhancing faults and extracted faults in the DEM.

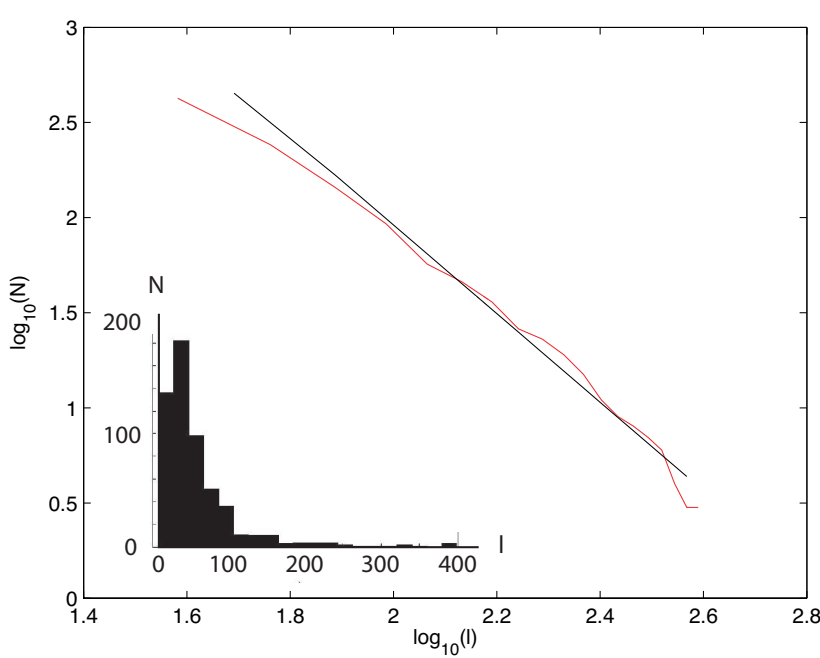

Fig. 6. Histogram of fault lengths (insert) and the corresponding cumulative histogram in logarithmic scale (red curve). The fractal dimension $(D=2.33)$ is estimated by fitting the distribution (black line, $\left.\mathrm{RMS}=0.045 ; R^{2}=0.99\right)$.

according to the following formula:

$N_{i}=\frac{C}{r_{i}^{D}}$

$N_{i}$ is the number of objects with a characteristic $r_{i}, C$ is a proportionality constant and $D$ is the fractal dimension. If $D$ is an integer it refers to the Euclidean dimension.

The length of faults is one (along with fault spacing, fault heaves etc.) of the characteristics that describes the fractal behavior of fault networks (Turcotte, 1997). Therefore

$N_{i}=\frac{C}{l_{i}^{D}}$ where $N_{i}$ is the number of faults having a length greater than $l_{i}, C$ is a proportionality constant and $D$ is the fractal dimension.

Taking logarithm on both sides of Eq. (5), we have

$\log \left(N_{i}\right)=\log (C)-D \log \left(l_{i}\right)$.

Once a satisfactory classification is achieved, we can use the notion of the object to determine the statistics of the fault populations. Every real world fault is an object in the image and the shape of the object can be used to find the approximate width and length of the faults. These statistics help us in determining the fractal dimension of the faults in that region. The lengths distribution and the corresponding fractal dimension $D \approx 2.35$ is calculated in the Magadi area using that information (Fig. 6). The linearity of the curve in the $\log -\log$ plot of the cumulative histogram of lengths of faults validates the method used in the present work. It also attests that the fault network is fractal.

The fractal dimension can also be calculated from the topology or the radar data of the region using the powerspectrum (PSD). The fractal dimension of a two-dimensional set can be calculated by calculating the slope of a linear fit plot of power $\mathrm{P}(\mathrm{k})$ vs. wavenumber, $\mathrm{k}$ on a log-log.

$S(k) \propto k^{-s}$

The PSD of a topologically 2-D remote sensing data can be calculated with 2-D Fourier transform. As described in Carr (1995) and Pentland (1984) the fractal dimension is calculated from the slope, $\mathrm{s}$ of the power versus frequency curve in logarithmic scale as:

$D=2.5-\frac{s}{2}$

The fractal dimension calculated using the PSD on $512 \times 512$ and $1024 \times 1024$ pixels subsets of the radar images and 
DEMs (example Fig. 7) corresponds to $D \approx 2.4$. The linear fits are remarkably good $\left(\mathrm{RMS}<0.05 ; R^{2}=0.99\right)$. This value does not vary for the whole fault network area.

The fractal dimension $(\mathrm{D}=2.4)$ can also be found using the variogram of $512 \times 512$ and $1024 \times 104$ pixels subsets the DEM (example Fig. 7). Linear fits are very good $\left(\mathrm{RMS}<0.04 ; R^{2}=0.99\right.$ ). Variogram analysis of radar images was not unequivocal due to high variance induced by speckle.

$\gamma\left(h_{i j}\right)=\frac{1}{2} E\left(\left[Y\left(x_{i}\right)-Y\left(x_{j}\right)\right]^{2}\right)$

$\gamma$ is the semi-variogram, $Y$ is the spatial function and $h_{i j}$ is the lag. The Hurst exponent $(\mathrm{H})$ is the slope of the variogram vs. lag on a log-log plot:

$\gamma\left(h_{i j}\right) \propto 2\left|h_{i j}\right|^{H}$

The fractal dimension is related to $\mathrm{H}$ by an empirical relation,

$D=2-\frac{H}{2}$

A fractal dimension very similar to that of the dimension of fault lengths is calculated using radar images PSDs and using DEM SVs and PSDs.

The similarity of fractal dimension shows that on images highly textured by faults, a direct fractal analysis is sufficient in order to asses the brittle strain regime. This shows that fractal dimension is a good indicator for describing complexity. We estimate the resolution to be less than $12 \mathrm{~m}$ (2 pixels) on Fine Beam SAR data and generated DEMs. Field and satellite images evidences suggest that the faults are highly segmented. The persistence of the linked segments in long, evolved faults imply that these segments must have a mechanical significance for the growth of faults. We quantitatively and qualitatively analyse the segment dimensions and morphology to study patterns of fault segment growth and linkage. Depending on the length of the faults and the number of segments then the morphology will change. A linked segment face has a typical almond shape, suggesting flexure of the brittle layer. For more evolved faults, the almond shape of the segments is conserved. The segmentation can be determined even in faults more than $10 \mathrm{~km}$ in length. The shapes of the flexures of the hangingwalls and of the accommodation zones between faults show that the faults grow by segment growth and linkage. After linkage, the flexures remain and are sometimes filled by isolated or connected lakes whose centers are localized on the zone where the individual segments have a maximum displacement, rather than the centre of the linked segments (fault) (e.g. Fig. 8).

\section{Fault model}

Using Fine Beam images we extract the morphology of the fault planes using transects along the hangingwalls and the footwalls (Fig. 8).

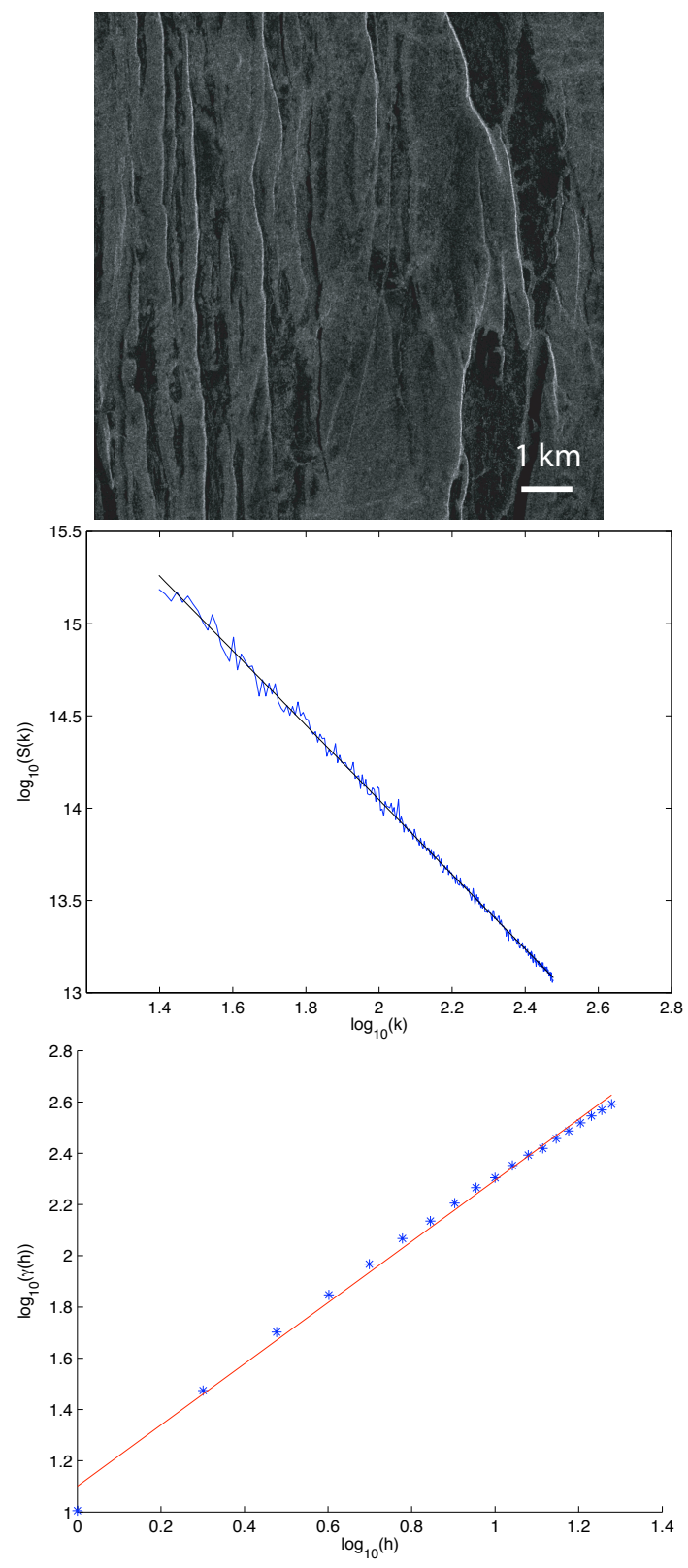

Fig. 7. top) Subset of a radar image (size $=512 \times 512$ pixels) middle) Calculating the fractal dimension from the slope of the power vs frequency of the radar image in logarithmic scale $(D=2.4$; fit: $\mathrm{RMS}=0.025 ; R^{2}=0.99$ ) bottom) Calculating the fractal dimension from the slope of the variogram vs. lag of the DEM in logarithmic scale $\left(\mathrm{D}=2.4\right.$; fit: $\left.\mathrm{RMS}=0.03 ; R^{2}=0.99\right)$.

Statistical analyses have been successfully applied in lowstrain regions where power-law scaling fits the data best (e.g., Scholz et al., 1991) and higher strain areas where exponential laws seem to describe the fault distribution (e.g., Cowie et al., 1993). This complexity arises from growth of faults, interaction and connection of faults to form networks. Based on this we propose that the fault network is an Iterated Function 


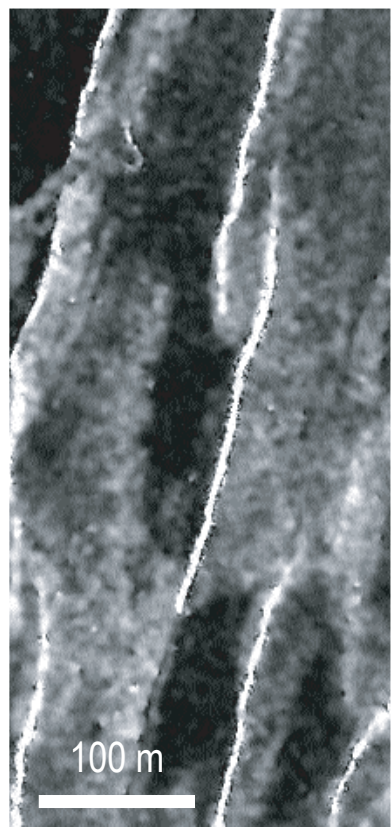

Fig. 8. Segmented faults imaged by Radarsat Fine Beam. This image also show the fault segmentation and the geometries of accommodations zones. On the left-hand sides of the faults, the image has a darker tone. This tone represents the thin coverage of lacustrine and eolian sediments that partly fill the depression created by concave flexures. Their attitude and smoothness will create specular reflection, thus darker tones. The footwall underlies a convex flexure that can also be seen by the grey variations caused by the change in amount of signal reflected across the footwall surface as the slope changes.

System (IFS) (Barnsley, 1988). Such a model allows us to understand the structural evolution of a basin in 4 dimensions, the fourth dimension being time. A model can now be proposed for describing fault growth.

Figure 9 shows a simple model explaining faulting mechanism. It starts with small segments whose dimensions are distributed according to a Gaussian law. In order to compensate the stress, the segments grow. In this process the growing segments connect forming complex systems which are fractal. Each fault nucleus has an equiprobable chance to occur at the surface. The first step is the synchronous appearance of nuclei sub-equally scattered all over the surface and respecting a given spacing, related to the zone of influence of each fault. The flexure of the foot-wall creates small depocenters. The growth of the depocenter, linked to the vertical displacement is late in respect to the longitudinal growth as can be seen on Fig. 8. The displacement is compensated by a longitudinal growth, not the opposite. As the segments grow longitudinally and perpendicular to the minimum compression stress vector (depending on the heterogeneity of the hosting rock), they enter the zone of influence of other segments and faults. The growth of each
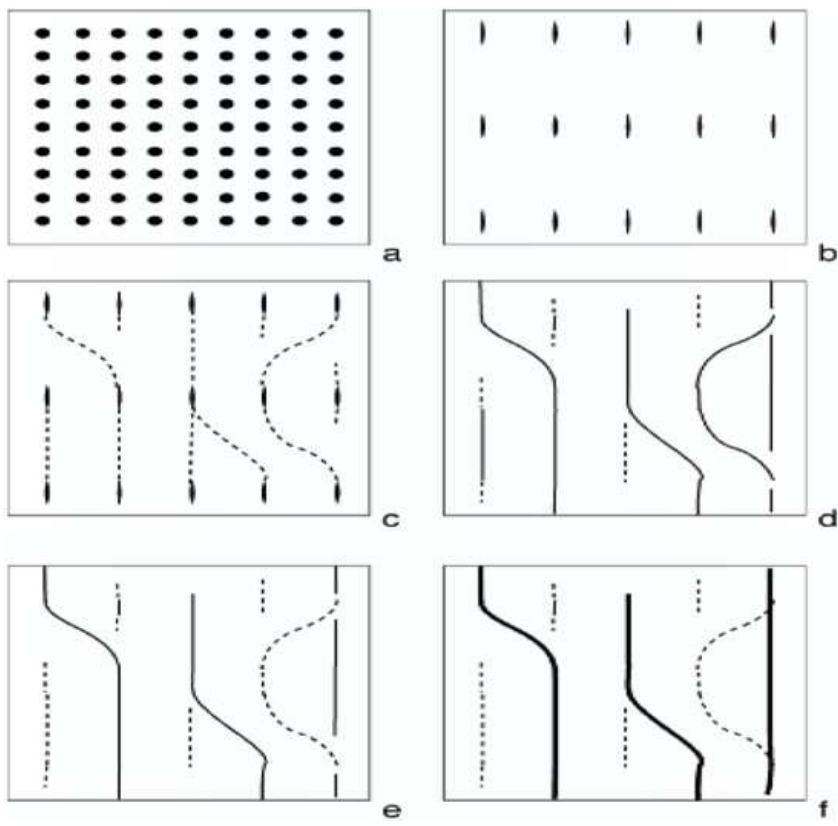

Fig. 9. Model of fault growth and interactions. See text for explanations.

segment is random (fractal) and the increment tends to create longer alignments. Small faults or segments that are offset from longer faults may be unfavourably orientated with respect to the stress patterns at propagating fault tips, and the fault growth ceases. The maximum horizontal displacement on one object being related to its size, the generation of long linear faults is preferred.The fractal dimension gives a measure of the complexity of the system.

\section{Conclusions}

We show that radar data and DEMs can be successfully classified using an object-based approach in order to map faults. This allow an unbiased and fast counting of faults for further statistical analysis. We also demonstrate that image texture is highly influenced by surface deformation, as shown by the identical fractal dimensions measured with SVs and PSDs. PSD and SV fractal dimension measurements attest the value of $\mathrm{D}=2.35$ found with box counting and cumulative lengths. The fractal analysis of the faults distribution reveals the importance of fractal dimension as a texture measure which can characterize the distribution.The fractal behavior allows us to propose an evolution model for the growth, interaction and linkage of faults in extensional domains. This model differs slightly from previous models where maximum fault lengths play a large role (e.g., Cowie and Scholz, 1992; Cowie et al., 1993; Marrett and Allmendinger, 1991; Scholz et al., 1991). In this model we consider faults as sets of connected segments. Even after hard linkage, the 
segments preserve mechanical properties. This affects our understanding of fault rupture during seismic events. It has been shown that earthquakes magnitudes are linearly related to fault ruptures and fault lengths in several geodynamic settings (Wells and Coppersmith, 1994). On the other hand, the earthquakes magnitudes are not related to fault lengths in the Kenya Rift (Seth et al., 2001) nor in the Main Ethiopian Rift (Keir et al., 2006). Both rifts are characterized by long faults and very low earthquake magnitudes. Our model relates seismic events to segment ruptures and thus allow us to reconcile surface deformation with measured seismicity. In low strain extensional rift, the deformation is diffuse and repartitioned on growing and linking segments. Based on this fractal behavior of faults we are now developing a numerical model of the extensional fault network evolution based on IFS and fitting the distribution of earthquakes magnitudes.

Acknowledgements. We are very grateful for the constructive critics from the editor, A. Tarquis and the careful reviews of C. Alonso, J. M. Redondo, T. Castellanos and an anonymous referee.

Edited by: A. Tarquis

Reviewed by: C. Alonso, M. T. Castellanos, J. M. Redondo, and another referee

\section{References}

Baker, B. H.: Geology of the Magadi area., Geol. Surv. Kenya Rep., 42, 82 p., 1958.

Baker, B. H., Mitchell, J. G., and Williams L. A. J.: Stratigraphy, geochronology and volcano-tectonic evolution of the KedongNaivasha-Kinangop region, Gregory Rift Valley, Kenya, J. Geol. Soc., London, 145, 107-116, 1988.

Barnsley, M.: Fractals everywhere, Academic Press, San Diego, pp 1-117, 1988.

Benz, U. C., Hoffmann, P., Willhauck, G., Lingenfelder, I., and Heynen, M.: Multi-resolution, object-oriented fuzzy analysis of remote sensing data for GIS-ready information, ISPRS Journal of Photogrammetry and Remote sensing, 58, 239-258, 2004.

Birt, C. S., Maguire, P. K. H., Khan, M. A., Thybo, H., Keller, G. R., and Patel, J.: The influence of pre-existing structures on the evolution of the southern Kenya Rift Valley-evidence from seismic and gravity studies, Tectonophysics, 248, 211-242, 1997.

Cardon, H.: Mécanisme de propagation des rseaux de failles: l'exemple du Rift Gregory (Kenya), Unpublished PhD thesis, Univ. Claude Bernard., 1999.

Carr, J. R.: Numerical Analysis for the Geological Sciences, Prentice Hall, Inc, NJ, 1995.

Castaing, C., Bourgine, B., Chiles, J. P., Genter, A., Ouilon, G., and Sornette, D.: Multiscale organization of joints and faults revealed by geostatistical, multifractal and wavelet techniques, in: EUG8. Terra Abstracts, Strasbourg, 1995.

Chu, D. and Gordon, G. R.: Evidence for motion between Nubia and Somalia along the Southwest Indian Ridge, Nature, 398, 6467, 1999.
Cowie, P. and Scholz, C. H.: Displacement-length scaling relationship for faults: data synthesis and discussion, J. Struc. Geol., 14(10), 1149-1156, 1992.

Cowie, P., Scholz, C. H., Edwards, M., and Malinverno, A.: Fault strain and seismic coupling on mid-ocean ridges, J. Geophys. Res., 98, 17 911-17 920, 1993.

Davy, P.: On the frequency-length distribution of the San Andreas fault system, J. Geophys. Res., 98(B7), 12 141-12 151, 1993.

Gloaguen, R., Rolet, J., Mouchot, M.-C., and Le Gall, B.: Geometry and role of transverse structures on the segmentation of the East African Rift System: Examples from the Kenya Rift, EOS, Trans. AGU, 80(46), 1024, 1999.

Gloaguen, R.: Analyse quantitative de l'extension continentale par imagerie satellitale et optique et radar. Application au rift sudkenyan, Unpublished PhD thesis, Univ. Bretagne Occidentale., 2000.

Henderson, F. M. and Lewis, A. J.: Principles and Applications of imaging Radar, manual of Remote sensing, 3rd edition, vol. 2., John Wiley and sons, 1998.

Keir, D., Stuart, G. W., Jackson, A., and Ayele, A.: Local Earthquake Magnitude Scale and Seismicity Rate for the Ethiopian Rift, Bulletin of the Seismological Society of America, vol. 96, no. 6, pp. 2221-2230, doi:10.1785/0120060051., 2006.

Marpu, P. R., Niemeyer, I., and Gloaguen, R.: A procedure for automatic object-based classification, Proceedings of the 1st International Conference on Object-based Image Analysis, 2006.

Marrett, R. and Allmendinger, R. W.: Estimates of strain due to brittle faulting: sampling of fault populations, J. Struct. Geol., 13, 735-738, 1991.

Mechie, J., Keller, G. R., Prodehl, C., Gaciri, S. J., Braile, L. W., Mooney, W. D., Gajewski, D. J., and Sandmeier, K. J.: Crustal structure beneath the Kenya Rift from axial profile data, Tectonophysics, 236, 179-199, 1994.

Nussbaum, S., Niemeyer, I., and Canty, M. J.: Feature Recognition in the Context of automated Object-Oriented Analysis of Remote Sensing Data monitoring the Iranian Nuclear Sites, Proceedings of Optics/Photonics in Security \& Defence, SPIE, 2005.

Pentland, A. P.: Fractal-Based Description of Natural Scenes, IEEE Trans. Pattern Anal. Machine Intell., 6, 661-674, 1984.

Scholz, C. H., Dawers, N. H., Yu, Y.-Z., Anders, M. H., and Cowie, P. A.: Fault growth and fault scaling laws: Preliminary results, J. Geophys. Res., 98, 21 951-21 961., 1991.

Seht, M. I., Blumenstein, S., Wagner, R., Hollnack, D., and Wohlenberg, J.: Seismicity, seismotectonics and crustal structure of the Southern Kenya Rift-new data from the Lake Magadi area. Geophysical Journal International 146, 2, 2001, p. 439-453, doi:10.1046/j.0956-540X.2001.01464.x., 2001.

Sella, G. F., Dixon, T. H., and Mao, A.: REVEL: A model for recent plate velocities from space geodesy, J. Geophys. Res., 107(B4), 2081, doi:10.1029/2000JB000033, 2002.

Turcotte, D. L.: Fractals and Chaos in Geology and Geophysics, 2nd Edition, Cambridge University Press, 1997.

Velde, B., Dubois, J., Touchard, G., and Badri, A.: Fractal analysis of fracture in rocks: The Cantor's Dust Method, Tectonophysics, 179, 345-352, 1990.

Velde, B. and Dubois, J.: Fractal analysis of fracture in rocks: The Cantor's Dust Method. Reply, Tectonophysics, 198, 112-115, 1991.

Villemin, T. and Sunwoo, C.: Distribution logarithmique self- 
similaire des rejets et longueurs de failles: exemple du Bassin Houiller Lorrain, C. R. Acad. Sci. Paris Série II(305), 1309$1312,1987$.

Watterson, J.: Fault dimensions, displacement and growth, Pageoph, 124, 365-373, 1986.
Wells, D. L. and Coppersmith, K. J.: New empirical relationships among magnitude, rupture length, rupture width, rupture area, and surface displacement, Bulletin - Seismological Society of America, 84(4), 974-1002, 1994. 